\title{
CLINICAL MYCOLOGY
}

\section{Oxygen requirements of Aspergillus species}

\author{
LINDA A. HALL* and D. W. DENNING* $†$ \\ * Department of Medicine, University of Manchester, Hope Hospital, Salford and $\dagger$ Department of Infectious Diseases \\ and Tropical Medicine (Monsall Unit), North Manchester General Hospital, Crumpsall, Manchester
}

\begin{abstract}
Summary. The growth of 24 Aspergillus isolates at low oxygen tensions was assessed. Isolates selected included A. fumigatus (10), A.terreus (6), A. niger (6), A. nidulans (1) and A.flavus (1). Three different agar media were used-potato dextrose agar (PDA), pH 5.6; brain heart infusion (BHI), $\mathrm{pH} 7 \cdot 4$; and a specially developed medium (Hall's) containing resazurinwith oxygen concentrations of $0,0 \cdot 025,0 \cdot 1,0.5$ and $2 \cdot 5 \%$. The $\mathrm{CO}_{2}$ concentration was $5 \%$. Agar plates were inoculated with $2 \times 10^{7}$ conidia $/ \mathrm{ml}$, loaded into jars and flushed with a special gas mixture at $37^{\circ} \mathrm{C}$. The plates were inspected at intervals of 3,5 and 10 days. On Hall's medium, none of the isolates grew at oxygen concentrations of 0 or $0.025 \%$, but $21(88 \%)$ of 24 grew at $0 \cdot 1 \%$. On PDA and BH1, all 14 isolates tested grew at oxygen concentrations of 0.5 and $2.5 \%$. Three of these 14 conidiated on PDA at oxygen $0.5 \%$ and 12 of 14 conidiated on PDA at oxygen $2.5 \%$. None grew without oxygen on these media. Thus, pathogenic Aspergillus spp. are capable of growth at low oxygen tensions, and this may have implications for pathogenicity and antifungal activity.
\end{abstract}

\section{Introduction}

Aspergilli are ubiquitous throughout the environment and are found in organic debris, decaying vegetation and soil. ${ }^{1}$ As causes of clinical infection, Aspergillus spp. are predominantly respiratory pathogens in immunocompromised hosts, but different host groups may also be susceptible to specific nonpulmonary disease (e.g., kidney disease in diabetic patients). ${ }^{2}$ Invasive aspergillosis is rare in immunocompetent hosts. The increase of immunocompromised patients and the greater degree of immune suppression within this group are responsible for a substantial increase in the incidence of this disease. As a proportion of all systemic mycoses found at autopsy, invasive aspergillosis now comprises $60 \%$, as compared to $17 \%$ a decade ago. ${ }^{3}$ The past decade has seen the emergence of new clinical entities caused by Aspergillus spp. and new host groups developing different forms of aspergillosis (e.g., pulmonary aspergillosis in patients with AIDS). ${ }^{4}$ Invasive aspergillosis is a devastating opportunist fungal disease with an unacceptably high mortality rate (in allogenic bone marrow transplant patients and cerebral aspergillosis it exceeds $94 \%$ ). ${ }^{5}$ It is difficult to diagnose infection in time for effective treatment to be given.

The most important single factor allowing anaerobic or micro-aerophilic organisms to flourish in body tissues is a low oxygen tension, which is usually the result of a compromised blood supply and poor tissue

Received 8 April 1994; revised version accepted 28 May 1994. $\dagger$ Correspondence should be sent to Dr D. W. Denning. perfusion. Typically, the pathological appearance of invasive aspergillosis shows infarcted tissue invaded by fungal hyphae. ${ }^{6}$ The aim of this study was to mimic these deep tissue conditions and establish the minimum oxygen requirements for growth of Aspergillus spp.

\section{Materials and methods}

\section{Aspergillus isolates}

Twenty-four Aspergillus isolates were selected: $A$. fumigatus (10), A. terreus (6), A. niger (6), A. nidulans (1) and A. flavus (1); most were clinical isolates. The inocula were prepared by culturing each isolate on

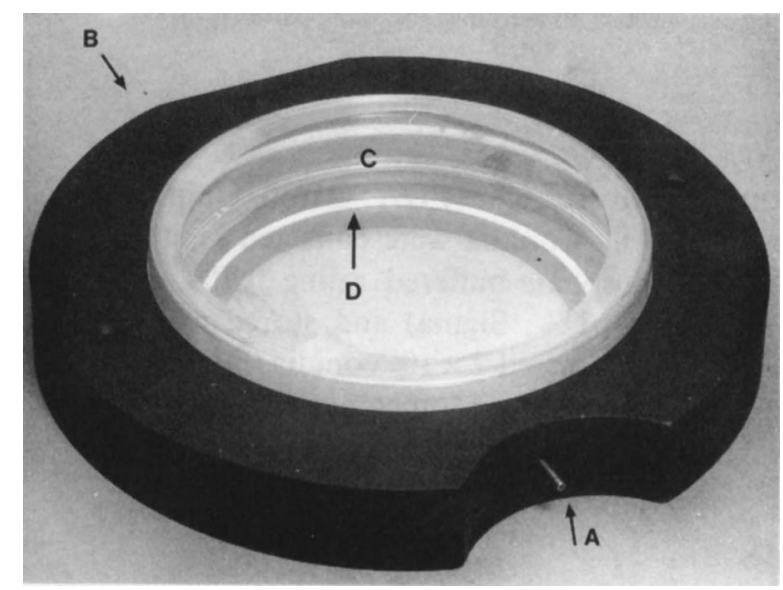

Fig. 1. Individual stacking ring with petri dish in position. A, inlet port; B, outlet port; C, peripheral seal; D, base seal. 


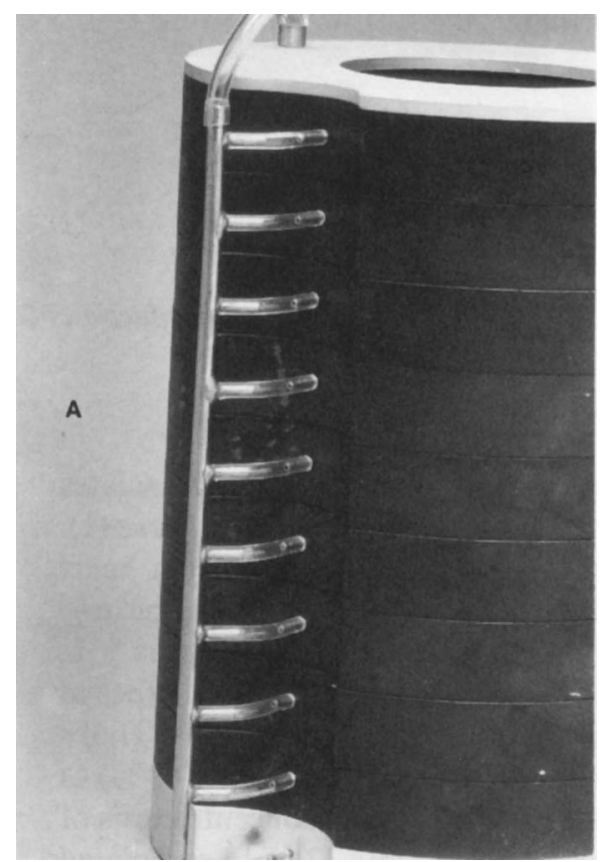

Fig. 2. Close-up of stacking insert and gas manifold. A. gas inlet manifold

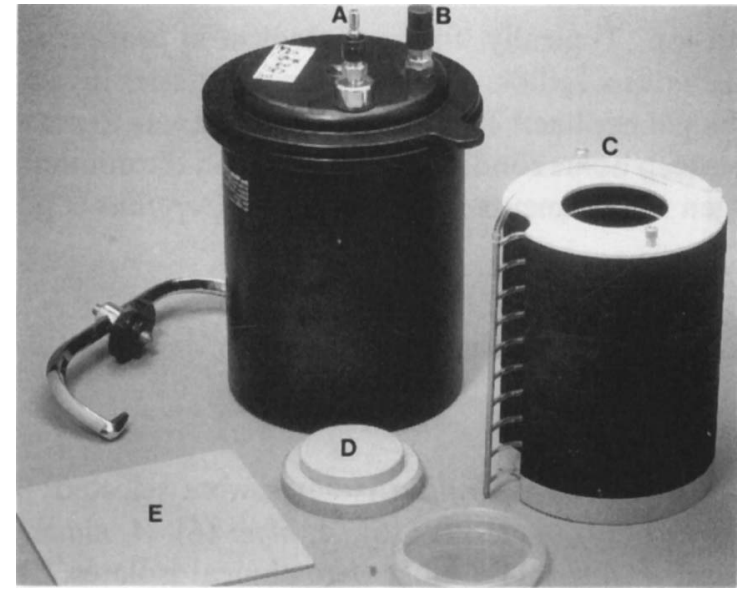

Fig. 3. Anaerobic jar showing modifications and accessories. A. sealed quick container (non-return inlet valve); B. pressure-relief valve: C. plate stacking insert with gas manifold; D. plate ejector or petri dish ejector; $E$, petri dish installing plate.

Potato Dextrose Agar (PDA; Oxoid/Unipath) for 10 days at $37^{\circ} \mathrm{C}$. Conidia were collected in sterile PBSTween (phosphate-buffered saline $0.9 \%$ containing Tween $800.01 \%$; Sigma) and stored at $4^{\circ} \mathrm{C}$. Each inoculum comprised $2 \times 10^{i}$ conidia $/ \mathrm{ml}$ quantified by counting in a haemocytometer.

\section{Media}

For testing. Brain Heart Infusion (BHI) Agar (Unipath), $\mathrm{pH} 7 \cdot 4, \mathrm{PDA}, \mathrm{pH} 5 \cdot 6$, and a modified defined medium (Hall's), pH 5, were used. Hall's medium contained (/L of distilled water): glucose $10 \mathrm{~g}$, yeast extract (Oxoid) $10 \mathrm{~g}, \mathrm{NaHCO}_{3} 6 \mathrm{~g}$, Lcysteine $\mathrm{HCl}$ (Sigma) $1 \mathrm{~g}, \mathrm{~K}_{2} \mathrm{HPO}_{4} 0.06 \mathrm{~g}, \mathrm{KH}_{2} \mathrm{PO}_{4}$ $0.06 \mathrm{~g},\left(\mathrm{NH}_{4}\right)_{2} \mathrm{SO}_{4} 0 \cdot 12 \mathrm{~g}, \mathrm{NaCl} 0 \cdot 12 \mathrm{~g}, \mathrm{MgSO}_{4} 0.012 \mathrm{~g}$ and $\mathrm{CaCl}_{2} 0.012 \mathrm{~g}$. The $\mathrm{pH}$ was adjusted to 5 with concentrated $\mathrm{HCl}$. Resazurin (Sigma) $0.02 \mathrm{~g} / \mathrm{L}$ and agar (Oxoid) to $1.8 \%$ were added. Resazurin is a reducing agent that is decolourised at low redox potential (e.g., $\leqslant-42 \mathrm{mV}$ ). ${ }^{7}$ The criterion for absolute anaerobiosis that is usually accepted for culture purposes is that a buffered resazurin solution at $\mathrm{pH} 7$ remains colourless. Hall's medium was colourless at pH 5 before and after autoclaving. The medium was heated to dissolve the agar and bubbled with $\mathrm{CO}_{2}$ to remove free $\mathrm{O}_{2}$. This was confirmed by a colour change from red to colourless of the resazurin indicator. It was then autoclaved at $115^{\circ} \mathrm{C}$ for $10 \mathrm{~min}$ and the lid was kept tightly closed. The medium remained colourless, at $\mathrm{pH} 5$ before and after autoclaving. The molten agar was then poured into petri dishes (previously stored in an anaerobic chamber) and allowed to cool.

\section{Incubation atmospheres}

The oxygen concentrations used were $0,0 \cdot 025,0 \cdot 1$, 0.5 and $2 \cdot 5 \%$. The $\mathrm{CO}_{2}$ concentration was $5 \%$. Gases were obtained from the British Oxygen Company, Morden and had quality assurance certificates. Specially adapted anaerobic jars were constructed (figs. 1-4), modified to ensure that the gas flow was uniform within each jar. Each agar plate was contained in two rubber O-Seal rings between which gas flowed. The gas was piped in and flowed evenly to each ring.

The poured plates were left in the anaerobic chamber for 2-5 days, then each was inoculated with $2 \times 10^{7}$ conidia with aseptic techniques in an atmosphere of $\mathrm{N}_{2} 85 \%, \mathrm{CO}_{2} 10 \%$ and $\mathrm{H}_{2} 5 \%$. Plates were sealed with paraffin and loaded into the modified anaerobic jars, transferred to a hot room at $37^{\circ} \mathrm{C}$ and continuously flushed with the special gas mixtures at a flow rate of $1 \mathrm{~L} / \mathrm{min}$ (fig. 4). The plates were inspected at intervals of 3,5 and 10 days in the anaerobic chamber and the presence or absence of growth was recorded. Plates that had shown no growth after 10 days were transferred to air at $37^{\circ} \mathrm{C}$ as a control to confirm a viable inoculum.

\section{Results}

The initial experiments were conducted with 14 isolates and PDA and BHI media at oxygen concentrations of $0,0 \cdot 5$ and $2 \cdot 5 \%$. None of the isolates grew at an oxygen concentration of $0 \%$ on either medium but all grew on transfer to air at $37^{\circ} \mathrm{C}$. All 14 isolates grew on PDA and BHI at oxygen $0.5 \%$ and $2.5 \%$; three of them conidiated in oxygen $0.5 \%$ and 12 of 


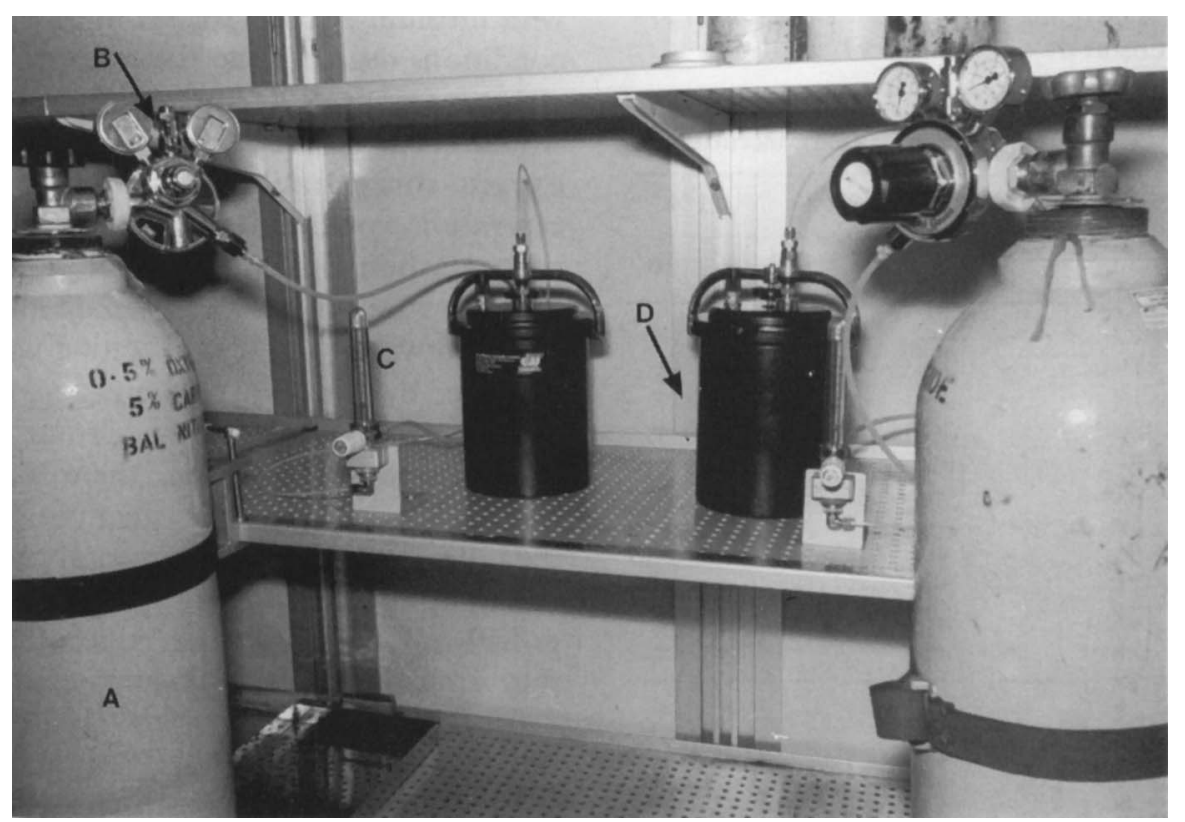

Fig. 4. Equipment in situ. A, high-pressure gas cylinder with specified gas mixture; B, high-pressure output regulator; C, flowmeter; D, anaerobic jar.

Table I. Growth of Aspergillus spp. on PDA and BHI at increasing oxygen concentrations

\begin{tabular}{|c|c|c|c|c|c|c|c|}
\hline \multirow{3}{*}{ Species } & \multirow{3}{*}{ Isolate no. } & \multicolumn{6}{|c|}{$\begin{array}{l}\text { Growth and conidiation (C) } \\
\text { at oxygen concentration }\end{array}$} \\
\hline & & \multicolumn{2}{|c|}{$0 \%$} & \multicolumn{2}{|c|}{$0.5 \%$} & \multicolumn{2}{|c|}{$2.5 \%$} \\
\hline & & PDA & BHI & PDA & BHI & PDA & BHI \\
\hline \multirow[t]{6}{*}{ A. fumigatus } & 10 & - & - & + & + & $+\mathrm{C}$ & + \\
\hline & 11 & - & - & $+\mathrm{C}$ & + & $+\mathrm{C}$ & + \\
\hline & 20 & - & - & $+\mathrm{C}$ & + & $+\mathrm{C}$ & + \\
\hline & 41 & - & - & + & + & $+\mathrm{C}$ & + \\
\hline & 71 & - & - & $+\mathrm{C}$ & + & $+\mathrm{C}$ & + \\
\hline & 72 & - & - & + & + & $+\mathrm{C}$ & + \\
\hline \multirow{3}{*}{ A. terreus } & 8 & - & - & + & + & $+\mathrm{C}$ & + \\
\hline & 10 & - & - & + & + & $+\mathrm{C}$ & + \\
\hline & 18 & - & - & + & + & $+\mathrm{C}$ & + \\
\hline \multirow[t]{3}{*}{ A. niger } & 1 & - & - & + & + & + & + \\
\hline & 4 & - & - & + & + & $+\mathrm{C}$ & + \\
\hline & 6 & - & - & + & + & $+\mathrm{C}$ & + \\
\hline A. nidulans & 2 & - & - & + & + & + & + \\
\hline A. flavus & 14 & - & - & + & + & $+\mathrm{C}$ & + \\
\hline
\end{tabular}

- , no growth by day $10 ;+$, growth by day $3 ;+\mathrm{C}$, growth and conidiation by day 3 .

them at $2.5 \%$ on PDA (table I). Growth was quite evident by day 3 and appeared to be more vigorous on PDA than BHI.

Hall's medium was then developed because of uncertainty about the concentration of dissolved oxygen in the standard media. Hall's medium was tested at oxygen $0.5 \%$ and by day 3 there was some change from colourless to purple. This colour change represents increasing dissolved oxygen concentration. Therefore, Hall's medium was tested at lower oxygen concentrations $(0.025$ and $0.1 \%)$. Each position in each jar was tested and no consistent variation in time to colour change was detected in any position in the jar.

On Hall's medium, there was no apparent growth on any plate by day 10 at oxygen $0.025 \%$ (table II). In oxygen $0 \cdot 1 \%, 16$ of 24 isolates showed varying degrees of growth by day 3. By day 5, 20 of 24 isolates had grown and 21 of 24 grew by day 10 . None of these isolates conidiated at oxygen $0 \cdot 1 \%$ on this medium.

Plates that exhibited no growth on Hall's medium after 10 days were incubated in air at $37^{\circ} \mathrm{C}$ and then at 
Table II. Growth of Aspergillus spp. on Hall's medium at low oxygen concentrations at day 3 and day 10

\begin{tabular}{|c|c|c|c|c|c|c|c|}
\hline \multirow{3}{*}{ Species } & \multirow{3}{*}{ Isolate no. } & \multicolumn{6}{|c|}{ Growth at oxygen concentration } \\
\hline & & \multicolumn{2}{|c|}{$0 \%$} & \multicolumn{2}{|c|}{$0.025 \%$} & \multicolumn{2}{|c|}{$0.1 \%$} \\
\hline & & 3 & 10 & 3 & 10 & 3 & 10 \\
\hline \multirow{10}{*}{ A. fumigatus } & 10 & - & - & - & - & + & + \\
\hline & 11 & - & - & - & - & + & + \\
\hline & 15 & - & - & - & - & + & + \\
\hline & 20 & - & - & - & - & + & + \\
\hline & 41 & - & - & - & - & + & + \\
\hline & 49 & - & - & - & - & + & + \\
\hline & 50 & - & - & - & - & - & + \\
\hline & 51 & - & - & - & - & - & - \\
\hline & 71 & - & - & - & - & + & + \\
\hline & 72 & - & - & - & - & + & + \\
\hline \multirow[t]{6}{*}{ A. terreus } & 4 & - & - & - & - & + & + \\
\hline & 6 & - & - & - & - & - & - \\
\hline & 8 & - & - & - & - & + & + \\
\hline & 10 & - & - & - & - & + & + \\
\hline & 17 & - & $*$ & $*$ & $*$ & - & + \\
\hline & 18 & - & - & - & - & - & + \\
\hline \multirow[t]{6}{*}{ A. niger } & 1 & - & - & - & - & - & + \\
\hline & 4 & - & - & - & - & + & + \\
\hline & 6 & - & - & - & - & + & + \\
\hline & 8 & - & - & - & - & - & + \\
\hline & 9 & - & - & - & - & + & + \\
\hline & 10 & - & - & - & - & - & - \\
\hline A. nidulans & 2 & - & - & - & - & + & + \\
\hline A. flavus & 14 & - & - & - & - & + & + \\
\hline
\end{tabular}

- , no growth; + , growth.

* Bacterial contamination

$4^{\circ} \mathrm{C}$. All isolates subsequently grew either at $37^{\circ} \mathrm{C}(22$ of 24$)$ or at $4^{\circ} \mathrm{C}$ ( 2 of 24$)$. This indicated that despite 10 days of oxygen deprivation and otherwise ideal germination conditions, the conidia were still viable and capable of germination.

\section{Discussion}

A new medium and test system were developed to assess the oxygen requirements of pathogenic $A s$ pergillus spp. with resazurin as an internal control for anaerobic conditions. The equipment was tested with the resazurin as an indicator dye and the positioning of the agar plates in the jars made no difference to the results. The concentration of oxygen was uniform throughout the jars and surface of the agar. The time taken for colouration of the medium was consistent with the different oxygen concentrations used- $\geqslant 5$ days at $0.025 \%$ and $\leqslant 3$ days at $0.5 \%$. The system with medium at constant $\mathrm{pH}$ and set up in anaerobic conditions decolourised the resazurin. This indicator changes to purple as the redox potential rises, which would occur before any substantial rise in dissolved oxygen concentration. ${ }^{8}$ Thus, surface oxygen concentration would have been higher than agar oxygen concentrations initially. Similar or identical oxygen concentrations in gas and agar are likely to have been achieved within 48-72 h of setting up the equipment, as judged by a medium colour change at higher oxygen concentrations, and possibly earlier at lower oxygen concentrations. Therefore, there would have been much oxygen flux in the system initially, but not over the latter part of the experimental period.

Pathogenic Aspergillus spp. appear to be capable of growth at very low oxygen concentrations. However, they are clearly not capable of anaerobic growth as defined by growth at oxygen $<0.01 \%{ }^{9}$ The growth rate is slowed at low oxygen concentrations and conidiation is delayed or absent. Conidiation varied with the medium used. Isolates that did not grow under experimental conditions subsequently grew when incubated in air, indicating that a low oxygen tension is not harmful to the conidia of Aspergillus spp. Thus oxygen can be considered an essential element for the growth but not survival of Aspergillus spp. These experiments did not allow determination of the absolute minimum oxygen requirement for growth.

The ability of aspergilli to continue to grow, albeit slowly, at low oxygen tensions has implications for pathogenicity. Aspergillus spp. are primarily pulmonary pathogens but different clinical and radiological patterns of disease are seen. Aspergilli often spread to the brain causing cerebral infarction. Different patterns of invasive aspergillosis could reflect differences between isolates in oxygen requirements, although only minor variation was detected in the isolates we tested. This variation might have clinical significance, although the actual concentrations of oxygen in infarcted tissue in invasive aspergillosis are not known. Other determinants of pathogenicity are likely to affect clinical patterns of aspergillosis and low oxygen concentration may affect, for example, the secretion of extracellular virulence factors. Whether susceptibility to antifungal agents varies at different oxygen concentrations is not known.

We are indebted to the Medical Physics Department, Hope Hospital, for constructing the agar stacking and gas flow arrangements in the anaerobic jars, and also to the Microbiology Department at Hope Hospital for the use of their facilities, to Dr Michelle Lawrence for her advice and helpful suggestions and to Mrs Julie Young for typing the manuscript.

\section{References}

1. Bardana EJ. The clinical spectrum of aspergillosis. Part I. Epidemiology, pathogenicity, infection in animals and immunology of Aspergillus. CRC Crit Rev Clin Lab Sci $1980 ; 13: 21-83$

2. Bodey GP, Vartivarian S. Aspergillosis. Eur $J$ Clin Microbiol Infect Dis $1989 ; 8: 413-437$.

3. Groll A. Shah PM, Menzel C. Just G, Schneider M, Hübner K.
Invasive mycosis in post-mortem findings. Trends in Invasive Fungal Infections II, Manchester, September 2-4, 1993; abstract no. 1.

4. Denning DW, Follansbee S, Scolaro M, Norris S, Edelstein D, Stevens DA. Pulmonary aspergillosis in AIDS. $N$ Engl $J$ Med 1991; 324: 654-662.

5. Denning DW, Stevens DA. Antifungal and surgical treatment 
of invasive aspergillosis: review of 2,121 published cases. Rev Infect Dis 1990; 12: 1147-1201.

6. Denning DW, Ward PN, Fenelon LE, Benbow EW. Lack of vessel wall elastolysis in human invasive pulmonary aspergillosis. Infect Immun 1992; 60: 5153-5156.

7. Visser W, Scheffers WA, Battenburg-Van Der Vegte WH, Van Dijken JP. Oxygen requirements of yeasts. Appl Environ Microbiol 1990; 56: 3785-3792.
8. Wimpenny JWT. The effect of $E_{h}$ on regulatory processes in facultative anaerobes. Biotech Bioengin 1969; 11 : 623-629.

9. Park MK, Myers RAM, Marzella L. Oxygen tensions and infections: Modulation of microbial growth, activity of antimicrobial agents, and immunologic responses. Clin Infect Dis 1992; 14: 720-740. 